\title{
Fiction Supporting Decelerated Expansion of the Universe
}

\author{
Umesh Kumar \\ (Student, VLSI \& ESD, Delhi Technological University, India)
}

\begin{abstract}
It has always been a topic of interest whether Universe is expanding or collapsing, if anyone of them is happening then whether the phenomena is accelerating or decelerating. This paper is a fiction supported by the basic principles of physics and Nebula hypothesis.
\end{abstract}

Keywords: Nebula Theory, Supernova, Gravity.

\section{INTRODUCTION}

The Big Bang theory is the prevailing cosmological model that explains the early development of the Universe. According to the Big Bang theory, the Universe was once in an extremely hot and dense state which expanded rapidly. This rapid expansion caused the young Universe to cool and resulted in its present continuously expanding state. According to the most recent measurements and observations, this original state existed approximately 13.7 billion years ago, which is considered the age of the Universe and the time the Big Bang occurred.

In my opinion it is hard to say whether the universe is expanding or collapsing because only of the observation of a few supernovae and their rate of expansion can never predict the complete nature of the Universe. So here we go for a fiction like explanation of the above mentioned phenomena.

\section{BEFORE BIG BANG}

It might have happened that before the Big-Bang, the system would have looked like a super-giant sun with extremely super-high temperature that could have ever been imagined. Now one question may be asked how such a system could be stable. The answer lies in the present Universe system's sun stability. It has been observed that big suns (stars) have higher temperature compared to smaller suns. It seems like the stability, mass and temperature of a star are parameters of a single equation (like the following one)

$$
S=f(T, M)=a \cdot T^{b} . M^{-c}
$$

Where $\mathrm{S}$ is stability factor, $\mathrm{T}$ is temperature and $\mathrm{M}$ is the mass of the star. $\mathrm{a}, \mathrm{b}$ and $\mathrm{c}$ are constants (or can be variables dependent of few more nature's parameters). If the mass and the temperature of the star are unbalanced, the star will not be able to survive i.e. less mass and high temperature will create a supernova and less temperature and high mass will turn system to go cold into a system like planets.

\section{AFTER BIG BANG}

After the super-explosion (The Big-Bang), according to the nebula hypothesis [1], the solar system began as a nebula, an area in the Milky Way Galaxy that was a swirling concentration of cold gas and dust. Due to some perturbation, possibly from the nearby supernova this cloud of gas and dust began to condense (fig. 1), or pull together under the force of its own gravity. Condensation was slow at first, but increase in speed as more material was drawn towards the centre of the nebula. This made gravity stronger, making condensation faster. This theory was justified by a well know experiment in which astronomers used a transparent glass tube of water with dissolved salt crystals (mingled in spaces). It was observed that salt crystals started to condense with many locality of condensation region. During the Nebulae formation the whole system still kept expanding with a very high velocity.

\section{REASON FOR DECELERATED EXPANSION}

According to my view nebula theory is not just only for the dust particles and gases created during the supernovae but can also be applied for the whole Universe system where all the galaxies are tiny particles in comparison of the center of the super-explosion (the big-bang). It may be possible that one day the expansion will stop and the whole universe will collapse into a single Star (sun or a super-nebula) and once again big-bang will occur and again the universe will be formed. 


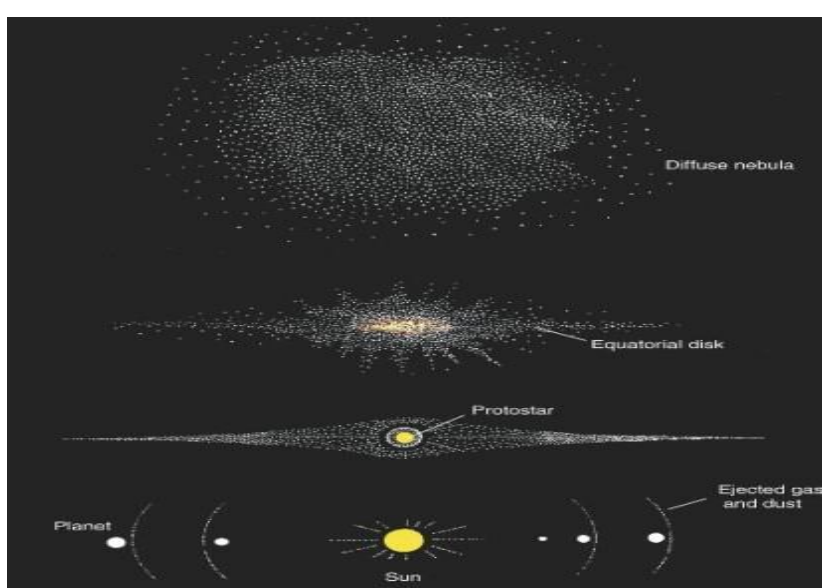

Fig.1. Nebula formation

History repeats itself.

\section{CONCLUSION}

\section{REFERENCES}

[1] Swedenborg, Emanuel (1734). (Principia) Latin: Opera Philosophica et Mineralia (English: Philosophical and Mineralogical Works).

[2] Woolfson, M.M. (1993). "Solar System - its origin and evolution". Q. J. R. Astr. Soc. 34: 1-20. Bibcode 1993QJRAS..34....1W. For details of Kant's position, see Stephen Palmquist, "Kant's Cosmogony Re-Evaluated", Studies in History and Philosophy of Science 18:3 (September 1987), pp.255-269.

[3] Kokubo, Eiichiro; Ida, Shigeru (2002). "Formation of protoplanet systems and diversity of planetary systems". The Astrophysical Journal 581 (1): 666-680. 